\title{
Adverse event burden and mechanical circulatory support: Looking toward the future
}

\author{
Francis D. Pagani, MD, PhD
}

\footnotetext{
From the Department of Cardiac Surgery, University of Michigan Health System, Ann Arbor, Mich. Disclosures: F.D.P. participates in contract research with HeartWare, Inc, and Thoratec Corporation that is administered by the University of Michigan.

Received for publication Sept 11, 2015; accepted for publication Sept 11, 2015; available ahead of print Oct 10, 2015 .

Address for reprints: Francis D. Pagani, MD, PhD, Department of Cardiac Surgery, 5161 Cardiovascular Center, SPC 5864, University of Michigan Health System, 1500 E Medical Center Dr, Ann Arbor, MI 48109 (E-mail: fpagani@umich.edu).

J Thorac Cardiovasc Surg 2016;151:10-2

$0022-5223 / \$ 36.00$

Copyright $(c) 2016$ by The American Association for Thoracic Surgery

http://dx.doi.org/10.1016/j.jtcvs.2015.09.052
}

A significant milestone in the field of mechanical circulatory support has been the introduction of durable continuous-flow (CF) left ventricular assist devices (LVADs) for the treatment of cardiogenic shock and advanced stage heart failure (HF). CF technology has enabled smaller device designs with markedly improved durability that have translated into significant clinical benefits, including improvements in survival, functional status, and quality of life and reduction in adverse events relative to older pulsatile pumps. ${ }^{1}$ These attributes of CF technology have resulted in a significantly greater adoption of mechanical circulatory support therapy by the medical community, which has extended this therapy from patients with cardiogenic shock or profound HF dependent on inotropes (Interagency Registry of Mechanically Assisted Circulatory Support [INTERMACS] levels 1-3) to patients who are ambulatory and non-inotrope dependent (INTERMACS level 4). ${ }^{2}$ Despite the significant advances in CF technology, these pumps have been associated with important adverse events, including pump thrombosis, stroke, bleeding, and device-related infection, ${ }^{2-4}$ and have caused unanticipated alterations in human biology (eg, acquired von Willebrand disease) that are related in part to shear stresses of the pump or reduced pulsatility. A recent safety communication (August 5, 2015) issued by the US Food and Drug Administration has highlighted adverse events with durable LVADs and emphasized the importance of restricting device use to currently approved populations to justify the current benefit and risk profile. ${ }^{5}$ The issue of adverse events with the current generation of pumps also contributed to the recent National Heart Lung and Blood Institute's decision to terminate the Randomized Evaluation of VAD Intervention Before Inotropic Therapy (REVIVE-IT) trial in March 2015 for futility as a result of changes in clinical equipoise in study investigators caused, in part, by concern regarding adverse events in study devices considered for the trial. REVIVE-IT was a prospective, multicenter, controlled, randomized clinical

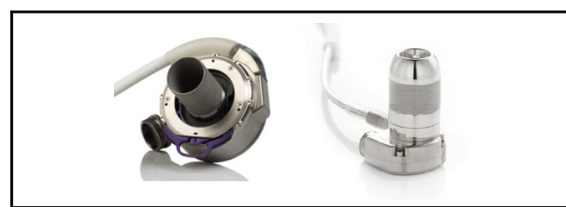

New generation of left ventricular assist devices entering clinical trial evaluation.

Central Message

Recent clinical trials with new durable LVADs are attempting to address the magnitude of device-related adverse event burden.

See Articles page 177, 192, 201, 213, 219,230 , and page 1352 in the November issue.

See Editorial Commentaries page 190, 199, 211, 217, 228, and 236.

trial investigating the use of initial LVAD therapy (HeartMate II; Thoratec Inc, Pleasanton, Calif) versus optimal medical management (OMM) for ambulatory patients not dependent on inotropes and ineligible for heart transplant, with New York Heart Association (NYHA) functional class III limitations (INTERMACS levels 5-7).

Two important clinical trials, Risk Assessment and Comparative Effectiveness of Left Ventricular Assist Device and Medical Management (ROADMAP) and A Clinical Trial to Evaluate the HeartWare Ventricular Assist System (ENDURANCE), have provided further insight on the issue of adverse event burden, and both were presented in April 2015 at the International Society of Heart and Lung Transplantation Annual Scientific Meeting in Nice, France. 6,7 ROADMAP was a prospective, multicenter, nonrandomized, controlled, observational study designed to evaluate the effectiveness of initial treatment with LVAD versus OMM, with later transition to LVAD therapy if necessary, in ambulatory patients with NYHA class IIIB or IV limitations who were not dependent on inotropes and who met the current US Food and Drug Administration-approved indications for destination therapy. The survivals at 1 year were $80 \%$ for patients assigned to initial LVAD support and $64 \%$ for patients initially assigned to OMM who did not transition to LVAD support at a later time. At 1 year, 39\% of patients assigned to initial LVAD support increased their walking 
distance by $75 \mathrm{~m}$, compared with $21 \%$ of patients assigned to OMM who did not transition to LVAD support. More patients in the group receiving initial LVAD had improvement to NYHA functional class I or II at 1 year $(77 \%$ vs $29 \%$ for those initially treated with OMM). Importantly, pump thrombus occurred in only $1.1 \%$ at 90 days and in $6.4 \%$ at 1 year. Both LVAD recipients and those assigned initial OMM had a 30-day mortality of only $1 \%$. Overall survival at 1 year was similar between those receiving initial LVAD therapy and initial OMM therapy when including those in the OMM group who received an LVAD at a later time. There were improvements in quality of life and a reduction in the severity of depression in patients receiving initial LVAD support. The overall risk of adverse events, including stroke, was higher in the group initially treated with LVAD support.

ENDURANCE was a prospective, randomized clinical trial evaluating newer $\mathrm{CF}$ technology with centrifugal design (HeartWare HVAD; HeartWare Corporation, Miami, Fla) against CF technology with axial design (HeartMate II) for destination therapy. The primary end point of the trial was survival evaluated at 2 years free of disabling stroke (modified Rankin score $\geq 4$ ) and on the originally implanted device. Importantly, the study demonstrated noninferiority of the CF technology with centrifugal design to the CF pump with axial design with respect to the primary end point. ${ }^{8}$ There was a higher risk of pump exchange, explant, or need for urgent transplant in the CF pump with axial design group (16.2\% vs $8.8 \%$ ) but a higher risk of stroke in the CF pump with centrifugal design group (28.7\% vs $12.1 \%)$.

The results of the ROADMAP and ENDURANCE trials attempted to address important issues in the field, but the conclusions in each trial were tempered by adverse event findings. Although the rate of pump thrombosis in ROADMAP was impressively low, the benefits of applying LVAD therapy in a population with less advanced stages of HF were clouded by the occurrence of adverse events and hospital readmissions in the group receiving initial LVAD support. LVAD therapy was not a clear and decisive winner in ROAPMAP, and this will require HF specialists to carefully weigh benefits versus risks in a patient population for which LVADs have not been widely adopted (INTERMACS level 4). ENDURANCE, although establishing the efficacy and safety of an alternative device for destination therapy, identified a concerningly high rate of stroke for the study device, increasing the complexity of future recommendations for device selection to patients. This finding prompted a supplementary study, ENDURANCE II, which is a prospective, randomized clinical trial again evaluating a CF pump with centrifugal design to a CF pump with axial design to investigate the influence of an improved blood pressure management algorithm with the CF pump with centrifugal design on all-cause stroke at 1 year (study enrollment completed August 2015). ${ }^{9}$

Although the burden of adverse events is troublesome, the validity of mechanical circulatory support therapy for treatment of advanced stage HF is clearly established and as a result will likely continue to stimulate a growing infusion of research investment and new technology into the field. ${ }^{10}$ New technology and not improvements in patient management has contributed to the largest gains in survival and reduction in adverse event burden during the past 2 decades. Important issues to address with future device designs include the following: (1) improved deviceblood compatibility to address thromboembolism and bleeding; (2) improved device software algorithms enabling pulsatility and variable speed control to address aortic insufficiency, bleeding, and functional capabilities; (3) smaller device designs enabling less-invasive surgical implantation or percutaneous insertion; (4) pediatric applications; (5) partial support technology; and (6) totally implantable designs that use wireless energy transfer systems. Device designs that significantly reduce the current rates of stroke and pump thrombosis would likely enable expansion of device therapy into the larger segment of patients with NYHA functional class III limitations or INTERMACS levels 5 and 6 . To this end, a number of important new devices have recently reported first-inhuman studies, with new pivotal trials underway or planned for later in 2015 . $^{11,12}$ Schmitto and colleagues ${ }^{12}$ recently reported first-in-human implantation of a new CF pump with centrifugal design and complete magnetic levitation and rotation of the internal rotor (HeartMate 3; Thoratec). Potential advantages of this new technology include large gaps between rotor and pump housing and sintering of internal pump surfaces, which could potentially translate into improved blood compatibility. Important control algorithms include introduction of a pulse. Pivotal trial evaluation of the HeartMate 3 began in the United States in late 2014 with the MOMENTUM 3 IDE Clinical Study Protocol. ${ }^{13}$ This trial is a prospective, multicenter, nonblinded, randomized study comparing the HeartMate 3 with the HeartMate II for advanced stage HF. The MVAD (HeartWare) is a CF pump with axial design and magnetic and hydrodynamic levitation of the internal rotor. The potential of the MVAD pump is a low-shear stress design of the internal impeller that may improve device-blood compatibility. In addition, the MVAD system incorporates a pulsatility algorithm to enhance aortic valve opening, which may affect the development of late aortic insufficiency and gastrointestinal bleeding. First-in-human implantations for the European CE Mark trial were recently reported. ${ }^{11}$

The current burden of adverse events has significantly limited application of LVAD therapy to broader populations of patients with less advanced stages of HF. Investment in 
new technology, however, has created great enthusiasm and expectation in the field that is likely to translate into important clinical benefits for our patients.

\section{References}

1. Slaughter MS, Rogers JG, Milano CA, Russell SD, Conte JV, Feldman D, et al; HeartMate II Investigators. Advanced heart failure treated with continuous-flow left ventricular assist device. N Engl J Med. 2009;361:2241-51.

2. Kirklin JK, Naftel DC, Pagani FD, Kormos RL, Stevenson LW, Blume ED, et al. Sixth INTERMACS annual report: a 10,000-patient database. J Heart Lung Transplant. 2014;33:555-64.

3. Kirklin JK, Naftel DC, Kormos RL, Pagani FD, Myers SL, Stevenson LW, et al. Interagency Registry for Mechanically Assisted Circulatory Support (INTERMACS) analysis of pump thrombosis in the HeartMate II left ventricular assist device. J Heart Lung Transplant. 2014;33:12-22.

4. Starling RC, Moazami N, Silvestry SC, Ewald G, Rogers JG, Milano CA, et al. Unexpected abrupt increase in left ventricular assist device thrombosis. $N$ Engl J Med. 2013;370:33-40.

5. Food and Drug Administration. Serious adverse events with implantable left ventricular assist devices (LVADs): FDA safety communication. Silver Spring, MD: US Food and Drug Administration. Available at: http://www.fda.gov/ MedicalDevices/Safety/AlertsandNotices/ucm457327.htm. Accessed August 5, 2015.

6. Pagani FD, Milano CA, Tatooles AJ, Bhat G, Slaughter MS, Birks EJ, et al. HeartWare HVAD for the treatment of patients with advanced heart failure ineligible for cardiac transplantation: results of the ENDURANCE destination therapy trial. J Heart Lung Transplant. 2015;34(4 Suppl):S9.

7. Estep JD, Starling RC, Horstmanshof DA, Rogers JG, Selzman CH, Shah KB, et al. Risk assessment and comparative effectiveness of left ventricular assist device and medical management in ambulatory heart failure patients (ROADMAP). J Heart Lung Transplant. 2015;34(4 Suppl):S80.

8. Pagani FD, Milano CA, Tatooles AJ, Bhat G, Slaughter MS, EJ Birks, et al. HeartWare HVAD for the treatment of patients with advanced heart failure ineligible for cardiac transplantation: results of the ENDURANCE destination therapy trial. Presented at: 2015 International Society of Heart and Lung Transplantation Annual Scientific Meeting; April 15-18, 2015; Nice, France. Available at: http://ir.heartware.com/phoenix.zhtml?c=187755\&p=irol-event Details\&EventId $=5188781$.

9. ClinicalTrials gov. A Clinical Trial to Evaluate the HeartWare Ventricular Assist System (ENDURANCE SUPPLEMENTAL TRIAL). ClinicalTrials.gov registration number NCT01966458. Bethesda, MD: National Library of Medicine; 2013. Available at: https://clinicaltrials.gov/ct2/show/NCT01966458. Accessed August 15, 2015.

10. Walker J. St. Jude Medical to buy Thoratec for $\$ 3.4$ billion. The Wall Street Journal. July 22, 2015. Available at: http://www.wsj.com/articles/st-jude-medicalto-buy-thoratec-for-3-4-billion-1437563728. Accessed August 15, 2015.

11. PR NewsWire. HeartWare International announces first human implants of the MVAD System in CE Mark international clinical trial. New York: PR NewsWire; July 20, 2015. Available at: http://www.prnewswire.com/news-releases/ heartware-international-announces-first-human-implants-of-the-mvad-systemin-ce-mark-international-clinical-trial-300115392.html. Accessed August 15, 2015.

12. Schmitto JD, Hanke JS, Rojas SV, Avsar M, Haverich A. First implantation in man of a new magnetically levitated left ventricular assist device (HeartMate III). J Heart Lung Transplant. 2015;34:858-60.

13. ClinicalTrials.gov. MOMENTUM 3 IDE Clinical Study Protocol (HM3). ClinicalTrials.gov registration number NCT02224755. Bethesda, MD: National Library of Medicine; 2014. Available at: https://clinicaltrials.gov/ct2/show/ NCT02224755. Accessed August 15, 2015. 